\title{
The trials and travels of tRNA
}

\author{
Sandra L. Wolin ${ }^{1,3}$ and A. Gregory Matera ${ }^{2}$ \\ ${ }^{1}$ Departments of Cell Biology and Molecular Biophysics and Biochemistry, Howard Hughes Medical Institute, Yale \\ University School of Medicine, New Haven, Connecticut 06536-0812 USA; ${ }^{2}$ Department of Genetics, Center for Human \\ Genetics and Program in Cell Biology, Case Western Reserve University and University Hospitals of Cleveland, \\ Cleveland, Ohio 44106-4955 USA
}

For relatively small molecules, the biogenesis of functional mature tRNAs is an amazingly complicated process. All tRNAs are transcribed initially as precursors containing 5' leader and $3^{\prime}$ trailer sequences that must be removed by processing. Pre-tRNAs also undergo a complex set of base modifications that are carried out by a series of enzymes that recognize specific features of tRNA structure. In addition, eukaryotic tRNAs have CCA added to their $3^{\prime}$ ends by a specialized nucleotidyltransferase. A subset of eukaryotic pre-tRNAs also contain intervening sequences, which are removed by a dedicated set of tRNA splicing enzymes. Lastly, tRNAs must be exported from the nucleus to the cytoplasm, and must undergo aminoacylation to participate in protein synthesis. Although the basic features of the tRNA biogenesis pathway have been appreciated for at least a decade, work in a number of laboratories over the last several years has resulted in several novel and unexpected insights into this complex process. For example, it was revealed recently that tRNAs are recognized and exported to the cytoplasm by a specialized export receptor, and that recognition by this export receptor is part of a quality control mechanism that ensures that incompletely processed and mutant RNAs will be retained in the nucleus. Furthermore, recent evidence suggests that certain of the various tRNA processing steps take place in discrete subcellular compartments, such that at least some steps of the tRNA biogenesis pathway are spatially as well as temporally ordered.

The eukaryotic tRNA biogenesis pathway is best understood in the budding yeast Saccharomyces cerevisiae, primarily due to the ease of combining genetics with biochemistry in this organism. However, virtually all components that have been identified in yeast have counterparts in higher cells, as would be expected for such a highly conserved process. Therefore, in this review, we emphasize recent results from yeast but also draw upon data from both prokaryotes and higher eukaryotic species. We provide an update on what is presently known about the processing events and paths taken by eukaryotic tRNAs following termination of

${ }^{3}$ Corresponding author.

E-MAIL sandra.wolin@yale.edu; FAX (203) 737-1761. transcription, culminating in their export to the cytoplasm. For more extensive descriptions of the enzymology of end-maturation, nucleotide modification, and tRNA splicing, see the following excellent reviews: Hopper and Martin (1992); Westaway and Abelson (1995); Grosjean et al. (1997); and Abelson et al. (1998).

\section{Binding by the La protein is required for the normal pathway of tRNA end maturation}

In eukaryotes, the first protein that binds to all newly synthesized pre-tRNAs is the La autoantigen, a highly abundant nuclear phosphoprotein. The La protein binds to the $3^{\prime}$ terminus of the newly synthesized transcript and protects this end from digestion by exonucleases (Yoo and Wolin 1997; Fan et al. 1998; Lin-Marq and Clarkson 1998). The La protein-bound pre-tRNA is the substrate for the ribonucleoprotein enzyme RNase P, which removes the 5 ' leader sequence by a single endonucleolytic cleavage (for review, see Frank and Pace 1998). Following removal of the $5^{\prime}$ leader, an as-yet-unidentified endonuclease removes the $3^{\prime}$ trailer $\left(\mathrm{O}^{\prime}\right.$ Connor and Peebles 1991; Furter et al. 1992; Yoo and Wolin 1997). This sequence of events is diagramed in Figure 1.

Although this pathway is followed by the majority of tRNAs in wild-type yeast cells (Yoo and Wolin 1997), both the order of events and the mechanism of 3 '-end removal can be altered without affecting the formation of functional tRNA. In both S. cerevisiae and Schizosaccharomyces pombe, the La protein is encoded by a dispensable gene. Interestingly, both the mechanism of $3^{\prime}$ end trimming and the order in which it occurs is altered in yeast cells that lack the La protein. In the absence of bound La protein, the first processing event is the nibbling of the 3' -end by exonucleases (Van Horn et al. 1997; Yoo and Wolin 1997). This exonucleolytic digestion halts at a stem formed by base-pairing between the purine-rich 5' leader sequences and pyrimidine-rich 3' trailers (Evans and Engelke 1990; Yoo and Wolin 1997). Following cleavage of the 5 ' leader sequence by RNase P, further exonuclease digestion generates the fully trimmed 3' end. Despite these differences in the pathway of tRNA end maturation, cells lacking the La protein contain approximately equal amounts of mature 
Figure 1. A schematic view of a yeast cell showing the pathway followed by an introncontaining pre-tRNA. The intervening sequence is shown in red, and the $5^{\prime}$ and $3^{\prime}$ extensions are black. After transcription, the newly synthesized pre-tRNA is immediately bound by the La protein (yellow oval). As described in the text, end maturation can occur in either the nucleolus or nucleoplasm. In our model, the $3^{\prime}$ trailer is removed by an endonuclease; however exonuclease digestion can also generate the mature 3' end (Yoo and Wolin 1997 and references therein). Similarly, although end maturation precedes intron removal in the model shown, this order can be reversed (see text). Note that certain nucleotide modifications (asterisks) take place on the newly synthesized pre-tRNA, others are added after end-trimming, and some occur only after removal of the intron. In our model, splicing, aminoacylation and binding by exportin-t (green oval) occur at the nuclear periphery. Binding of certain tRNAs uncharged tRNA by Arclp is not shown but is described in the text.

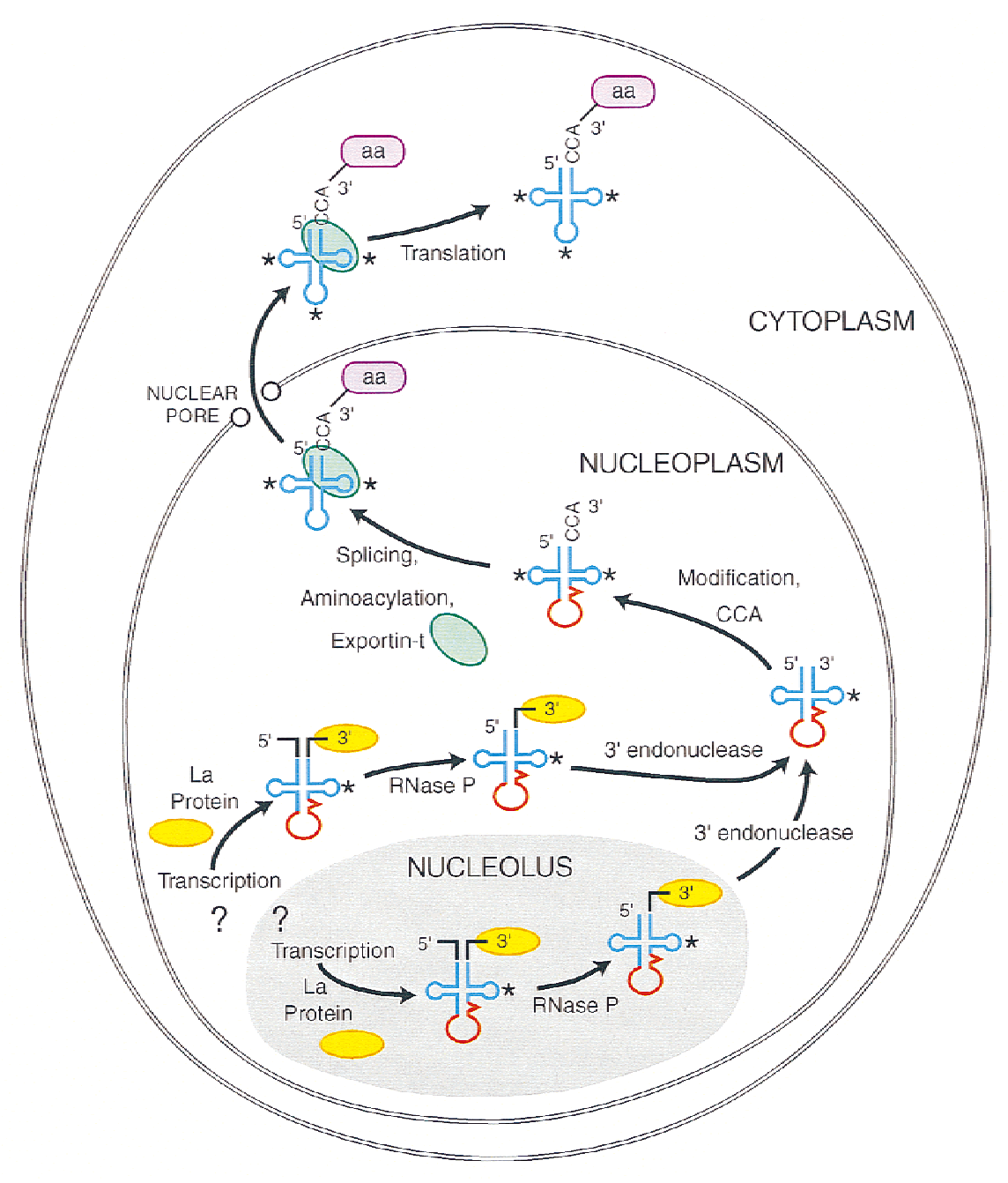

tRNA. Thus, as described in prokaryotes (Li and Deutscher 1996), yeast cells contain redundant mechanisms for processing tRNA 3' ends.

Despite the finding that wild-type pre-tRNAs do not require the La protein for processing, binding by La may facilitate certain aspects of the maturation process. $S$. cerevisiae cells require the La protein for growth when a point mutation disrupts the anticodon stem of an essential pre-tRNA ${ }_{\mathrm{CGA}}^{\mathrm{Ser}}$. As introduction of a second mutation that restores base-pairing in the stem eliminates the requirement for the La protein, the La protein may function as an 'RNA chaperone' to promote the formation of the correctly folded pre-tRNA structure (Yoo and Wolin 1997). Because addition of unphosphorylated, but not phosphorylated, human La protein to processing reactions containing yeast RNase $\mathrm{P}$ was found to inhibit removal of the 5' leader sequence, it has been proposed that phosphorylation of the La protein could regulate tRNA processing (Fan et al. 1998). Although tRNA maturation is not known to be a regulated process, $\mathrm{La}$ protein bound to the $3^{\prime}$ trailer could influence either the accessibility of the $5^{\prime}$ leader or the rate of cleavage by RNase P.
For those tRNAs that contain intervening sequences, either end maturation or splicing can occur first. In $S$. cerevisiae, intermediates corresponding to the 'end maturation first' pathway are much more abundant than those corresponding to the 'splicing first' pathway, suggesting that for most tRNAs, end maturation usually precedes splicing (Lee et al. 1991; O'Connor and Peebles 1991; Yoo and Wolin 1997). However, for particular tRNAs, the "splicing first" intermediates become more prominent when yeast are grown at high temperature, suggesting that the order of processing for at least some pre-tRNAs can be influenced by growth conditions (O'Connor and Peebles 1991). Consistent with this idea, it was recently demonstrated that the pathway that predominates in Xenopus oocyte injection experiments depends upon the amount of pre-tRNA introduced into the nucleus (Lund and Dahlberg 1998). When low amounts of pre-tRNA ${ }^{\mathrm{Tyr}}$ (or DNA encoding pre-tRNA ${ }^{\mathrm{Tyr}}$ ) were injected, splicing of the intron occurred prior to end maturation. However, in the presence of higher concentrations of pre-tRNA, end-maturation preceded splicing, presumably because the splicing machinery became saturated (Lund and Dahlberg 1998). 


\section{Does end maturation take place in the nucleolus as well as the nucleoplasm?}

Surprisingly, it appears that the 5' tRNA maturation step occurs not only in the nucleoplasm (as might have been expected), but also in the nucleolus. Bertrand et al. (1998) used fluorescent deoxyoligonucleotides complementary to the introns of several yeast pre-tRNAs to localize the precursors in the nucleus. Such probes will detect all three forms of intron-containing precursors present in yeast (see Fig. 1) but should not detect mature tRNA. In one experiment, probes complementary to the introns of two different pre-tRNAs, Leu3 and Trp, were combined and used for in situ hybridization to $S$. cerevisiae cells. Remarkably, the two combined intron probes localized almost exclusively to the yeast nucleolus (Bertrand et al. 1998). As expected, a probe that only detected the mature spliced tRNA was primarily cytoplasmic.

One potential criticism of this type of experiment is that the tRNA probes might detect the excised introns rather than the actual site of processing. Most of the introns excised from pre-tRNA are stable enough to be detected in Northern analyses $\left(\mathrm{O}^{\prime}\right.$ Connor and Peebles 1991). Furthermore, a significant fraction of the introncontaining pre-tRNA in cells has already undergone end maturation (O'Connor and Peebles 1991). In this case, the probes would be detecting not the site of end maturation, but the site of either intron excision or degradation. However, these possibilities were ruled out by Bertrand et al. (1998) in a second set of experiments, in which they used a probe complementary to the spacer region between two tRNAs that are always transcribed as a single unit. Similar to what they had observed with the combined intron probes, the probe against the dimeric pre-tRNA localized to the nucleolus. The two tRNAs that make up the dimeric transcript, tRNA ${ }^{\mathrm{Arg}}$ and tRNA ${ }^{\text {Asp }}$, lack introns, thus ruling out the possibility that the probe was detecting the site of intron excision. Furthermore, after RNase P cleavage separates the downstream tRNA, tRNA ${ }^{\text {Asp }}$, from tRNA ${ }^{\text {Arg }}$ and the transcribed spacer, $3^{\prime} \rightarrow 5^{\prime}$ exonucleases degrade the spacer region from tRNA ${ }^{\text {Arg }}$ to generate the mature $3^{\prime}$ end. Thus, the spacer sequence does not accumulate in the cell (Engelke et al. 1985).

Because the first step in processing for all these tRNAs is removal of the $5^{\prime}$ leader sequence by RNase P, Bertrand et al. (1998) also examined the subcellular location of this enzyme. Using two different in situ hybridization probes, the RNA component of RNase $\mathrm{P}$ was found to be predominantly nucleolar. However, both probes also detected several discrete sites of hybridization within the nucleoplasm, well removed from the nucleolus (Bertrand et al. 1998). Thus, for some pre-tRNAs, 5' maturation may occur in the nucleoplasm.

Support for the idea that some tRNAs undergo end trimming in the nucleoplasm rather than the nucleolus comes from similar experiments in which a probe directed against the intron of pre-tRNA $\mathrm{IUAU}_{\mathrm{UI}}^{\mathrm{II}}$ was used to detect this pre-tRNA in situ (Sarkar and Hopper 1998). In these experiments, the fluorescent signal was uniformly distributed throughout the nucleus. This probe, like the intron probes discussed above, will also detect the excised intron (which at 60 nucleotides is the longest intron in $S$. cerevisiae) as well as the intron-containing precursor that has already undergone end trimming (see Fig. 1). Thus, in such experiments, it is uncertain whether the observed signal reflects the site of end maturation, tRNA splicing, or intron degradation. Interestingly, the intron-specific signal became more intense when cells containing the temperature sensitive rna1-1 mutation were examined at the nonpermissive temperature (Sarkar and Hopper 1998). This gene encodes the GTPase-activating protein (RanGAP1) that stimulates GTP hydrolysis by Ran/TC4, the ras family member required for nuclear transport (Becker et al. 1995; Corbett et al. 1995). In cells containing the rna1-1 mutation, intron-containing pre-tRNAs accumulate at the nonpermissive temperature (Hopper et al. 1978; Knapp et al. 1978). Thus, the fact that the nuclear signal increases under these conditions makes it likely that the probe is detecting intron-containing tRNAs, rather than the excised intron. Nonetheless, since the majority of introncontaining pre-tRNAs that accumulate in rna1-1 cells have already undergone end maturation, the observed signal may not reflect the location of the end-trimming machinery.

If at least some yeast tRNAs undergo end maturation in the nucleolus, might this also be true in higher eukaryotes? Attempts to localize RNase P in mammalian cells have given conflicting results. In one set of in situ hybridization experiments, using both antisense oligonucleotides and nick-translated DNA probes, RNase P RNA was localized to the cytoplasm and the nucleolus in several human cell lines, as well as to structures at the edge of the nucleolus known as perinucleolar compartments (PNCs) (Matera et al. 1995; Lee et al. 1996). As these PNCs also contain certain other RNAs transcribed by RNA polymerase III, they are thought to represent sites at which some aspect of RNA biogenesis or export occurs (Matera et al. 1995). One intriguing possibility is that the PNCs, by analogy to the recent data from yeast, represent the site of tRNA maturation. However, antibodies directed against a protein component of the RNase P known as the Th or To autoantigen failed to detect PNCs (Lee et al. 1996), suggesting that the fraction of RNase P RNA that is found in PNCs is not assembled into the mature RNP. Furthermore, in a separate set of in situ hybridization experiments in normal rat kidney cells, Jacobson et al. (1997) reported that RNase P was located throughout the nucleoplasm, that is, not confined to the nucleolus.

Given the conflicting results from both yeast and mammalian cells, more localization experiments clearly are needed. In yeast, localization of each of the 10 families of intron-containing pre-tRNAs should reveal whether the majority of these pre-tRNAs are indeed located within the nucleolus. In mammals, the vast majority of pre-tRNAs lack intervening sequences, making it more difficult to specifically localize pre-tRNAs in these cells. Nonetheless, as all human tRNA ${ }^{\text {Tyr }}$ genes 
contain introns (van Tol and Beier 1988), it should be possible to localize at least these pre-tRNAs within nuclei. Because most protein components of RNase $\mathrm{P}$ are also shared by the related nucleolar endonuclease MRP (mitochondrial RNA processing) (Morrissey and Tollervey 1995; Stolc and Âltman 1997; Chamberlain et al. 1998), it has not been possible to use antibodies directed against protein components of RNase P to localize this enzyme in yeast or mammalian cells. However, a recent purification of RNase $P$ from yeast cells has revealed that one of the nine protein subunits, Rpr2p, is unique to RNase P (Chamberlain et al. 1998). It should therefore be feasible, using either antibodies to Rpr2p or epitopetagged yeast strains, to determine if the fully assembled ribonucleoprotein complex is localized within the nucleolus.

If maturation of at least some pre-tRNAs occurs in the nucleolus, how do the transcripts get there? One possibility is that tRNA transcription occurs in the nucleolus. Although the site(s) of tRNA gene transcription have not been experimentally determined, the fact that the genes encoding tRNAs are scattered throughout most eukaryotic genomes makes this prospect, although not impossible, at least somewhat unlikely. Alternatively, if transcription of tRNA genes occurs in the nucleoplasm, how might pre-tRNAs travel to the nucleolus? The only protein that is known to bind newly synthesized, unprocessed pre-tRNA is the La autoantigen. Since a fraction of the La protein has been localized to the nucleolus in mammalian cells (Deng and Tan 1985; Graus et al. 1985), it is conceivable that binding by the La protein could form part of a signal for nucleolar localization. For this reason, coupled with the fact that the order of events and mechanism of $3^{\prime}$ end maturation is altered in yeast cells lacking the La protein, it would be interesting to determine if pre-tRNAs remain nucleolar in yeast strains that lack the La protein.

\section{Many nucleotide modifications, as well as CCA addition, take place in the nucleoplasm prior to intron removal}

It has been known for many years that some modified nucleotides are added to newly synthesized pre-tRNAs, others are added to the end-trimmed but intron-containing species, and still others occur only after removal of the intervening sequence (Nishikura and De Robertis 1981). Because end maturation, intron removal and CCA addition all occur in the nucleus (Melton et al. 1980), it has long been assumed that those modifying enzymes that modify the various intron-containing precursors will be localized in the nucleus, and that enzymes that modify the fully spliced tRNA will be located in the cytoplasm (for review, see Maden 1998).

With the identification of some of these modifying enzymes in yeast, it has been possible to test these predictions directly. For example, the enzyme $N^{2}, N^{2}$-dimethylguanine tRNA methyltransferase, encoded by the gene TRM1 in S. cerevisiae, is able to catalyze the modification of G26 in many tRNAs to $\mathrm{m}_{2}^{2} \mathrm{G} 26$ (Ellis et al. 1986).
This modification occurred largely on the end-matured but unspliced pre-tRNA when the processing of yeast tRNA $^{\text {Tyr }}$ was examined in Xenopus oocytes (Nishikura and De Robertis 1981). As would be expected for an enzyme that acts primarily on otherwise mature but unspliced pre-tRNA, Trm1p is found largely at the nuclear periphery where the tRNA splicing machinery is also located (Rose et al. 1995; see below). Similarly, the enzyme Pus1p, (pseudouridine synthase 1), which catalyzes the conversion of uridine to pseudouridine in three distinct regions of the tRNA (Motorin et al. 1998), is localized within the nucleus (Simos et al. 1996b). This is consistent with the observation that certain of the pseudouridylations carried out by this enzyme were absolutely dependent on the presence of the intervening sequence in the pre-tRNA (for review, see Grosjean et al. 1997).

In the case of certain other modifying enzymes, the experimentally determined locations have been contrary to expectations. For example, the formation of isopentenyl adenosine at position 37 of some tRNAs was found to occur only on pre-tRNAs that had already undergone splicing (Nishikura and De Robertis 1981; Spinelli et al. 1997). However, the yeast enzyme that catalyzes the formation of $i^{6} \mathrm{~A}, \operatorname{Mod} 5 \mathrm{p}$, has been localized throughout the cell, in the nucleus, cytosol, and mitochondria (where it catalyzes $i^{6} \mathrm{~A}$ formation for mitochondrial tRNAs; Boguta et al. 1994). Recently, the nuclear portion of Mod5p was shown to be concentrated in the nucleolus (Hunter et al. 1999). Experiments in which the nuclear localization signal was mutated to cause the majority of the enzyme to be mislocalized to the cytosol revealed that the cytosolic form of Mod5p was capable of efficiently modifying $i^{6} \mathrm{~A}$ (as judged by the efficiency of tRNA $\mathrm{UAA}_{\mathrm{TA}}^{\mathrm{Ty}}$ in carrying out nonsense suppression; Hunter et al. 1999|. One possibility is that pre-tRNAs that lack intervening sequences are modified by the nucleolar form of Mod5p, whereas intron-containing pre-tRNAs would undergo $i^{6} \mathrm{~A}$ modification in the cytoplasm, following intron removal. Alternatively, it is possible that the nucleolar Mod5p modifies other RNA species, although tRNAs are the only RNAs known to contain $i^{6} \mathrm{~A}$. Finally, as it was only possible to detect and localize Mod5p in yeast when MOD5 was overexpressed more than 10-fold on a high copy plasmid (Boguta et al. 1994; Hunter et al. 1999|, the observed nucleolar location could be a result of overproducing the protein.

Although most of the tRNA modifications studied to date are not essential for viability in S. cerevisiae, it was recently reported that one modification, the formation of 1-methyladenosine at position 58 of $\mathrm{tRNA}_{\mathrm{i}}^{\mathrm{Met}}$ is required for growth. In these experiments, a nuclear complex containing the essential Gcd10 and Gcd14 proteins was shown to be necessary for the formation of 1-methyladenosine modification on tRNA (Anderson et al. 1998). Although 18 yeast tRNAs contain $\mathrm{m}^{1} \mathrm{~A}$ at position 58 , the lethality of strains lacking Gcd10p could be complemented by genes encoding tRNA ${ }_{i}^{\text {Met }}$ on a high copy vector, indicating that modification of $\mathrm{tRNA}_{\mathrm{i}}^{\mathrm{Met}}$ is the only essential role of this protein in yeast (Anderson et al. 1998). Consistent with this idea, cells containing muta- 
tions in either GCD10 or GCD14 have reduced levels of mature tRNA $\mathrm{i}_{\mathrm{i}}^{\text {Met }}$ due to decreased stability and/or inefficient processiing of pre-tRNA $\mathrm{i}_{\mathrm{i}}^{\text {Met }}$ lacking this modification (Anderson et al. 1998). Interestingly, a recent purification of the methyltransferase that is responsible for modification of the unusual cap structure present on all trypanosomal mRNAs has revealed that two of the subunits appear to be homologs of Gcd10p and Gcd14p (C. Tschudi and E. Ullu, pers. comm.). Thus, the Gcd10p/ Gcd14p complex is highly conserved throughout evolution, and functions in the modification of other RNA molecules besides tRNAs.

Before leaving the nucleus, eukaryotic tRNAs also undergo the post-transcriptional addition of CCA to their 3' termini. The first evidence that CCA addition was a nuclear event came from examination of the intron-containing pre-tRNAs that accumulated in a yeast mutant strain defective in RNA export (Hopper et al. 1978; Corbett et al. 1995). In the mutant strain, these unspliced pre-tRNAs were found to contain CCA at their $3^{\prime}$ ends (Knapp et al. 1978, 1979). More recent experiments in which Ccalp, the yeast ATP(CTP):tRNA nucleotidyltransferase, was localized in cells revealed that this enzyme is found in three different subcellular compartments: the nucleus, cytosol, and mitochondria (Wolfe et al. 1996). Experiments in which the cytosolic fraction of Ccalp was mislocalized to the nucleus resulted in the accumulation of 3 ' shortened tRNAs in the cytosol, consistent with the idea that the cytoplasmic Ccalp functions in the repair of CCA termini (Wolfe et al. 1996).

\section{The cytoplasm or bust: tRNAs utilize a special export pathway}

Recent work from numerous laboratories has shown that macromolecular transport through the nuclear pore is mediated by saturable receptors that recognize specific signals on the various cargo molecules (for review, see Izaurralde and Adam 1998; Mattaj and Englmeier 1998; Weis 1998). Despite the differences in their various transport substrates, all nuclear import and export receptors thus far identified are members of the importin $\beta$ superfamily and bind to the small GTPase Ran (Fornerod et al. 1997; Görlich et al. 1997). Export of tRNA from the nucleus is no exception; mature tRNAs were shown recently to be recognized by a specialized receptor called exportin-t (Arts et al. 1998a; Kutay et al. 1998). Exportin-t was first identified in human cells by its ability to bind to immobilized Ran-GTP and by its homology to Loslp, a yeast member of the importin $\beta$ superfamily that had long been known to function in tRNA biogenesis (Arts et al. 1998a; Kutay et al. 1998; see below). As would be expected for a nuclear export receptor, experiments performed in both human cultured cells and Xenopus oocytes have revealed that exportin-t shuttles between the nucleus and cytoplasm (Arts et al. 1998a; Kutay et al. 1998). Although exportin-t binds directly to tRNA, its affinity for tRNA increases several hundredfold in the presence of RanGTP, indicating that the binding of RanGTP and tRNA is highly cooperative (Kutay et al. 1998). Direct proof that exportin-t functions in tRNA export was provided by experiments in which exportin-t was injected into Xenopus oocytes along with tRNA substrates. Coinjection of exportin-t increased the rate of tRNA export and alleviated the saturation of export that normally occurs in the presence of competitor tRNA (Arts et al. 1998a; Kutay et al. 1998).

Although the exportin-t/tRNA complex, like other transport receptor/cargo complexes, binds RanGTP, GTP hydrolysis by Ran does not appear to be required for tRNA export. Injection of a mutant form of Ran, RanQ96L, which is unable to hydrolyze GTP (Bischoff et al. 1994; Klebe et al. 1995) into Xenopus oocyte nuclei, blocks export of mRNA and snRNAs but not tRNAs (Izaurralde et al. 1997). However, the presence of RanGTP is required, as injection of a mutant form of Ran that is unable to stably bind GTP, RanT24N, inhibits export of all three classes of RNA (Izaurralde et al. 1997).

The yeast homolog of exportin-t is likely to be none other than Los1p (Hellmuth et al. 1998). Although Los1p was first described nearly 20 years ago as a mutation that caused yeast cells to accumulate end-trimmed, but unspliced pre-tRNAs (Hopper et al. 1980), the precise function of this protein in tRNA biogenesis had been mysterious. Similar to other transport receptors, Loslp was shown recently to bind the yeast RanGTP homolog Gsp1p (Hellmuth et al. 1998). As would be expected for a tRNA export receptor, the affinity of Loslp for GsplpGTP increased substantially in the presence of tRNA (Hellmuth et al. 1998). Furthermore, Loslp has been localized to the nuclear pore using indirect immunofluorescence (Simos et al. 1996b) and shown to physically interact with at least two nuclear pore proteins, Nsplp and Nup2p (Hellmuth et al. 1998).

Given that Los $1 p$ functions as a tRNA export receptor, it is curious that this protein is encoded by a dispensable gene in yeast (Hurt et al. 1987). Because tRNA export is likely to be essential for cell survival, yeast must have evolved redundant mechanisms to accomplish this process. One possibility is that one of the other, as-yet-uncharacterized, importin $\beta$ family members in yeast acts redundantly with Loslp to export tRNA to the cytoplasm. Alternatively, other tRNA-binding proteins may transport tRNA to the cytoplasm through a pathway normally used for protein export. Interestingly, LOS1 becomes essential in yeast strains that contain certain mutations in other components of the tRNA biogenesis pathway. Synthetic lethal interactions have been observed between mutations in LOS1 and mutations in TFC4, which encodes a polymerase III transcription factor, PUS1, which encodes a pseudouridine synthase, $A R C 1$, which encodes a tRNA-binding protein that functions in tRNA aminoacylation as well as GCD11, which encodes the $\gamma$-subunit of the translation initiation factor eIF2 (Simos et al. 1996a,b; Hellmuth et al. 1998). One explanation for the observed genetic interactions is that yeast cells are able to live with the reduced levels of cytoplasmic tRNA caused by mutations in LOS1. However, when cells lacking Los1p contain additional mutations that further decrease the levels of mature cytoplas- 
mic tRNA, the amount of functional tRNA available for protein synthesis drops below a critical threshold (Hellmuth et al. 1998). An alternative, but not necessarily exclusive, possibility is that one or more of these tRNAbinding proteins, such as Arclp or Gcd11p, might function redundantly with Los1p to export tRNA through a protein export pathway. Lastly, some of the observed genetic interactions may indicate that tRNA export interfaces with other steps in the tRNA biogenesis and protein synthesis pathways (see below).

\section{Intron removal and nucleotide modification may be coupled to tRNA export}

Analyses of transport mutants in S. cerevisiae have long provided tantalizing hints that pre-tRNA splicing and export may be linked. For example, the rna1-1 mutant, which was shown many years ago to accumulate endmatured but intron-containing pre-tRNAs (Hopper et al. 1978), is now known to contain a mutation in the yeast homolog of the protein RanGAP1, which is required for nuclear transport (Becker et al. 1995; Corbett et al. 1995). Similarly, cells lacking Los1p, the newly identified tRNA export receptor, accumulate unspliced tRNAs (Hopper et al. 1980), as do cells containing mutations in certain nuclear pore proteins (Sharma et al. 1996). These observations have been incorporated into a model in which tRNA splicing in yeast takes place at the nuclear pore complex (Clark and Abelson 1987; Tanner et al. 1988; Sharma et al. 1996).

In agreement with the notion that intron removal may accompany export, the machinery that removes intervening sequences from pre-tRNAs has been localized to the nuclear periphery. The tRNA splicing reaction consists of just three enzymes: an endonuclease that removes the intervening sequence, a ligase that joins the two half molecules leaving a 2 ' phosphate at the splice junction, and a phosphotransferase that transfers the $2^{\prime}$ phosphate to NAD (for review, see Westaway and Abelson 1995; Abelson et al. 1998). There is evidence that both the endonuclease and the ligase are associated with the nuclear envelope in yeast. Specifically, the endonuclease fractionates with membranes, but can be released with nonionic detergents (Peebles et al. 1983; Rauhut et al. 1990). Furthermore, the ligase was localized using immunoelectron microscopy to the inner nuclear membrane. Intriguingly, $>40 \%$ of the immunogold particles were located within $25 \mathrm{~nm}$ of a nuclear pore (Clark and Abelson 1987). Recently, the tRNA splicing endonuclease was purified and its four subunits cloned (Trotta et al. 1997). Of all the subunits, only one, Sen2p, contains a potential transmembrane sequence. Thus, Sen2p may anchor the endonuclease to the nuclear membrane (Trotta et al. 1997).

Genetic interactions in $S$. cerevisiae also hint at the possibility that certain tRNA modifications are linked to export. The pseudouridine synthase Puslp was identified originally because cells containing certain combinations of mutations in the nuclear pore protein Nsplp and the tRNA transport receptor Los1p required Pus1p for viabil- ity (Simos et al. 1996b). Furthermore, although cells lacking either Pus1p or Los1p are viable at all temperatures, cells lacking both proteins exhibit reduced growth at $30^{\circ} \mathrm{C}$ and are dead at $37^{\circ} \mathrm{C}$ (Simos et al. 1996b). One explanation for the observed genetic interactions is that the pseudouridylations carried out by Puslp may contribute to the recognition of transport-competent tRNA by the components of a redundant export pathway.

\section{Further trials: proofreading and nuclear aminoacylation}

It has been noted by numerous workers that mutant pretRNAs are processed inefficiently, tend to be less stable than wild-type pre-tRNAs, and are frequently retained in the nucleus (Melton et al. 1980; Zasloff et al. 1982; Traboni et al. 1984; Tobian et al. 1985; Haselbeck and Greer 1993; Boelens et al. 1995; Lund and Dahlberg 1998). Furthermore, wild-type pre-tRNAs containing immature $3^{\prime}$ and $5^{\prime}$ ends are not exported to the cytoplasm (Melton et al. 1980; Lund and Dahlberg 1998). Although the molecular mechanisms by which this 'nuclear proofreading' occurs are only beginning to be understood, inefficient recognition of mutant pre-tRNAs by the various processing enzymes is likely to contribute to the observed reduction in processing. In addition, proteins that bind to the unprocessed pre-tRNAs (such as the La protein, the various modification enzymes, and the pre-tRNA splicing machinery), may contribute to tRNA quality control by retaining immature tRNAs in the nucleus.

At least some of the proofreading of immature and mutant tRNAs was recently demonstrated to be due to discrimination by the tRNA export machinery. Human exportin-t has a 10-fold higher affinity for tRNAs isolated from cultured cells than unmodified, in vitro-transcribed tRNAs, indicating that at least some modifications are required for efficient recognition (Kutay et al. 1998). Recent experiments in which mutant forms of yeast tRNA $^{\text {Phe }}$ were assayed for exportin-t binding revealed that several mutations that altered tRNA structure also prevented exportin-t binding (Arts et al. 1998b). When export of these mutant tRNAs was assayed by microinjection into Xenopus oocytes, a good correlation was found between exportin-t binding and nuclear export. Likewise, tRNAs containing immature 5' or 3' ends were not bound by exportin-t (Arts et al. 1998b) nor exported from oocyte nuclei (Melton et al. 1980; Lund and Dahlberg 1998), indicating that recognition by exportin-t is an important part of the mechanism by which cells ensure that only functional, mature tRNAs reach the cytoplasm. Experiments in which chemical and enzymatic probes were used to analyze the features of tRNA structure recognized by exportin-t revealed that this protein interacts primarily with the acceptor and $\mathrm{T} \psi \mathrm{C}$ stems (Arts et al. 1998b). However, the fact that mature $5^{\prime}$ and $3^{\prime}$ ends are crucial for exportin-t recognition indicates that the protein also monitors tRNA ends (Arts et al. 1998b).

It was recently reported that tRNAs are subjected to what might be regarded as the ultimate proofreading step, aminoacylation, prior to export from the nucleus 
(Lund and Dahlberg 1998). In these experiments, several

${ }^{32} \mathrm{P}$-labeled pre-tRNAs were synthesized in vitro and injected into oocyte nuclei. After a short incubation, the labeled RNA in both the nuclear and cytoplasmic fractions was found to have undergone aminoacylation. The nuclear aminoacylation was not dependent upon tRNA export, as it occurred in oocytes in which transport was blocked by depleting the nuclear pool of RanGTP. Blockage of nuclear aminoacylation using specific inhibitors slowed (but did not prevent) export, suggesting that aminoacylated tRNAs are preferentially recognized by some component of the export machinery (Lund and Dahlberg 1998). Similarly, Arts et al. (1998b) found that a tRNA ${ }^{\text {Phe }}$ mutant that was unable to undergo aminoacylation was exported somewhat more slowly that the wild-type tRNA. Although experiments to determine if exportin- $t$ exhibits a higher binding affinity for aminoacylated tRNAs have not yet been reported, it is tempting to speculate that exportin-t also mediates the preferential export of these tRNAs.

\section{Arc1p: an adapter between the tRNA transport and mRNA translation machinery?}

Yet another tRNA-binding protein, known as Arclp, was identified through its genetic interactions with Los1p. Arclp is found in a complex with two aminoacyl-tRNA synthetases in S. cerevisiae, MetRS and GluRS, where it is required for the efficient aminoacylation of tRNA by these enzymes (Simos et al. 1996a, 1998). Although cells lacking Arc1p grow slowly, cells lacking both Arc1p and Los $1 p$ are inviable. Experiments in which Arclp was localized within cells using indirect immunofluorescence have revealed that while Arclp is cytoplasmic, the protein appears to be concentrated at the nuclear periphery (Simos et al. 1996a). This finding, together with the observed synthetic lethality with Los $1 \mathrm{p}$, have been incorporated into a model in which tRNA is delivered by Los $1 p$ to Arc1p which transfers it to the synthetases (Simos et al. 1996a, 1998).

How might this model be reconciled with the observation that, in Xenopus oocytes, aminoacylation occurs inside the nucleus and facilitates efficient tRNA export? One possibility is that, in both yeast and higher eukaryotes, aminoacylation takes place at the nuclear pore. This scenario would be consistent with the observed concentration of Arclp at the nuclear periphery in $S$. cerevisiae (Simos et al. 1996a). Alternatively, aminoacylation in the nucleoplasm may be confined to higher species. In organisms from Drosophila to humans, nine aminoacyl-tRNA synthetases associate with three other polypeptides to form a multienzyme complex (Filonenko and Deutscher 1994; Kerjan et al. 1994). One of the three nonsynthetase polypeptides, p43, was recently cloned and found to share a domain with Arclp, suggesting that it may be the mammalian homolog (Quevillon et al. 1997). Interestingly, p43, unlike the rest of the mammalian multisynthetase complex, is largely nuclear (Popenko et al. 1994). Thus, p43/Arc1p may exist in higher eukaryotes both as a free nuclear protein as well as a component of the multisynthetase complex. It is thus possible that, at least in higher eukaryotes, the nuclear fraction of $\mathrm{p} 43 /$ Arclp facilitates aminoacylation by nuclear tRNA synthetases.

Interestingly, although $S$. cerevisiae cells lacking $A R C 1$ grow slowly, these cells become inviable in the presence of mutations in either LOS1, which encodes the tRNA export receptor, or NSP1, which encodes a nuclear pore protein (Simos et al. 1996a 1998). One intriguing possibility is that, in both yeast and mammalian cells, binding by nuclear p43/Arc1p allows certain tRNAs to exit the nucleus through a protein export pathway that recognizes and transports p43/Arclp to the cytoplasm. The existence of these sorts of redundant mechanisms for tRNA nuclear transport would explain the puzzling result that Los $1 \mathrm{p}$ is encoded by a dispensable gene. Although Arclp is predominantly cytoplasmic in S. cerevisiae (Simos et al. 1996a), it is possible that the protein shuttles between the nucleus and the cytoplasm to facilitate export.

\section{Perspectives: does tRNA channeling begin at birth?}

Experiments performed in permeabilized mammalian cells have led to the proposal that during repeated cycles of protein synthesis, aminoacyl tRNA molecules are directly transferred, or 'channeled,' from the synthetases to the elongation factor EF-1 to the ribosome, and then are transferred back to the aminoacyl synthetases without dissociating into the surrounding medium (Stapulionis and Deutscher 1995). The discovery that certain tRNA binding proteins, such as Loslp and p43/Arclp, exhibit numerous biochemical and genetic interactions with other components of the tRNA modification, transport, aminoacylation, and protein synthesis pathways suggest that tRNAs are also channeled from one component to the next during the various steps in their complicated biogenesis. For example, Los $1 p$ physically interacts with Gcd11p, the $\gamma$ subunit of initiation factor eIF-2, in a two-hybrid assay (Hellmuth et al. 1998). One interpretation of this result might be that Los 1 , upon entering the cytoplasm, directly hands tRNA $\mathrm{A}_{\mathrm{i}}^{\text {Met }}$ to eIF-2 to facilitate efficient initiation of translation. Similarly, binding of certain tRNAs by $\mathrm{p} 43 /$ Arclp, whether it occurred in the nucleus or cytoplasm, could serve to deliver these tRNAs directly to aminoacyl synthetases (Quevillon et al. 1997; Simos et al. 1998).

If the channeling model of protein synthesis can be extended to include tRNA export from the nucleus, what about the earliest steps in tRNA biogenesis? To begin to answer this question, more information is clearly needed about these very early steps. For example, where in the nucleus are the different tRNA genes transcribed? Is binding by the La protein required for nucleolar targeting or retention of the nascent pre-tRNAs? If end maturation occurs in the nucleolus, how are the end-matured but intron-containing tRNAs transported to the nuclear periphery for splicing? It is our expectation that further biochemical, genetic, and cell biological analyses of the tRNA biogenesis pathway will continue to yield impor- 
tant insights, not only into the basic mechanisms of tRNA maturation and processing, but also into subcellular organization and RNA transport.

\section{Acknowledgments}

We are grateful to Chris Greer, Kazio Tycowski, and an anonymous reviewer for valuable comments on the manuscript. We also thank all the colleagues who sent us their unpublished manuscripts, and apologize to anyone whose work we may have inadvertently overlooked. The authors are supported by $\mathrm{Na}$ tional Institutes of Health grants R01-GM48410 (S.L.W.) and R01-GM53034 (A.G.M.). S.L.W. is an Assistant Investigator of the Howard Hughes Medical Institute.

\section{References}

Abelson, J., C.R. Trotta, and H. Li. 1998. tRNA splicing. J. Biol. Chem. 273: 12685-12686.

Anderson, J., M. Pak, L. Phan, B.A. Carlson, R. Cuesta, K. Asano, G.R. Bjork, M. Tamane, and A.G. Hinnebusch. 1998. The essential Gcd10p-Gcd14p nuclear complex is required for 1-methyladenosine modification and maturation of initiator methionyl-tRNA. Genes \& Dev. 12: 3650-3662.

Arts, G-J., M. Fornerod, and I.W. Mattaj. 1998a. Identification of a nuclear receptor for tRNA. Curr. Biol. 8: 305-314.

Arts, G-J., S. Kuersten, P. Romby, B. Ehresmann, and I.W. Mattaj. 1998b. The role of exportin-t in selective nuclear export of mature tRNA. EMBO J. (in press).

Becker, J., F. Melchior, V. Gerke, F.R. Bischoff, H. Ponstingl, and A. Wittinghofer. 1995. RNA1 encodes a GTPase-activating protein specific for Gsplp, the Ran/TC4 homologue of Saccharomyces cerevisiae. J. Biol. Chem. 270: 11860-11865.

Bertrand, E., F. Houser-Scott, A. Kendall, R.H. Singer, and D.R. Engelke. 1998. Nucleolar localization of early tRNA processing. Genes \& Dev. 12: 2463-2468.

Bischoff, F.R., C. Klebe, J. Kretschmer, A. Wittinghofer, and H. Ponstingl. 1994. RanGAP1 induces GTPase activity of nuclear Ras-related Ran. Proc. Natl. Acad. Sci. 91: 25872591.

Boelens, W.C., I. Palacios, and I.W. Mattaj. 1995. Nuclear retention of RNA as a mechanism for localization. RNA 1: 273283.

Boguta, M., L.A. Hunter, W.C. Shen, E.C. Gillman, N.C. Martin, and A.K. Hopper. 1994. Subcellular locations of MOD5 proteins: Mapping of sequences sufficient for targeting to mitochondria and demonstration that mitochondrial and nuclear isoforms commingle in the cytosol. Mol. Cell. Biol. 14: 2298-2306.

Chamberlain, J.R., Y. Lee, W.S. Lane, and D.R. Engelke. 1998. Purification and characterization of the nuclear RNase P holoenzyme complex reveals extensive subunit overlap with RNase MRP. Genes \& Dev. 12: 1678-1690.

Clark, M.W. and J. Abelson. 1987. The subnuclear localization of tRNA ligase in yeast. J. Cell Biol. 105: 1515-1526.

Corbett, A.H., D.M. Koepp, G. Schlenstedt, M.S. Lee, A.K. Hopper, and P.A. Silver. 1995. Rnalp, a Ran/TC4 GTPase activating protein, is required for nuclear import. J. Cell Biol. 130: $1017-1026$

Deng, J.S. and E.M. Tan. 1985. Effect of actinomycin D on the expression of nuclear antigen SS-B/La. J. Invest. Dermatol. 84: $225-228$

Ellis, S.R., M.J. Morales, J.M. Li, A.K. Hopper, and N.C. Martin. 1986. Isolation and characterization of the TRM1 locus, a gene essential for the $\mathrm{N}^{2}, \mathrm{~N}^{2}$-dimethylguanosine modification of both mitochondrial and cytoplasmic tRNA in Saccharomyces cerevisiae. J. Biol. Chem. 261: 9703-9709.

Engelke, D.R., P. Gegenheimer, and J. Abelson. 1995. Nucleolytic processing of a tRNA ${ }^{\mathrm{Arg}}$-tRNA ${ }^{\mathrm{Asp}}$ dimeric precursor by a homologous component from Saccharomyces cerevisiae. J. Biol. Chem. 260: 1271-1279.

Evans, C.F. and D.R. Engelke. 1990. Yeast extracts for transfer RNA gene transcription and processing. Methods Enzymol. 181: 439-450.

Fan, H., J.L. Goodier, J.R. Chamberlain, D.R. Engelke, and R.J. Maraia. 1998. $5^{\prime}$ processing of tRNA precursors can be modulated by the human La antigen phosphoprotein. Mol. Cell. Biol. 18: 3201-3211.

Filonenko, V.V. and M.P. Deutscher. 1994. Evidence for similar structural organization of the multienzyme aminoacyltRNA synthetase complex in vivo and in vitro. J. Biol. Chem. 269: 17375-17378.

Fornerod, M., J. van Deursen, S. van Baal, A. Reynolds, D. Davis, K. Gopal Murti, J. Fransen, and G. Grosveld. 1997. The human homologue of yeast CRM1 is in a dynamic subcomplex with CAN/Nup214 and a novel nuclear pore component Nup88. EMBO I. 16: 807-816.

Frank, D.N. and N.R. Pace. 1998. Ribonuclease P: Unity and diversity in a tRNA processing ribozyme. Annu. Rev. Biochem. 67: 153-180.

Furter, R., M. Snaith, D.E. Gillespie, and B.D. Hall. 1992. Endonucleolytic cleavage of a long $3^{\prime}$-trailer sequence in a nuclear yeast suppressor tRNA. Biochemistry 31: 1081710824.

Görlich, D., M. Dabrowski, F.R. Bischoff, U. Kutay, P. Bork, E. Hartmann, S. Prehn, and E. Izaurralde. 1997. A novel class of RanGTP binding proteins. J. Cell Biol. 138: 65-80.

Graus, F., C. Cordon-Cardo, E. Bonfa, and K.B. Elkon. 1985 Immunohistochemical localization of La nuclear antigen in brain. Selective concentration of the La protein in neuronal nucleoli. J. Neuroimmunol. 9: 307-319.

Grosjean, H., Z. Szweykowska-Kulinska, Y. Motorin, F. Fasiolo, and G. Simos. 1997. Intron-dependent enzymatic formation of modified nucleosides in eukaryotic tRNAs: A review. Biochimie 79: 293-302.

Haselbeck, R.C. and C. Greer. 1993. Minimum intron requirements for tRNA splicing and nuclear transport in Xenopus oocytes. Biochemistry 32: 8575-8581.

Hellmuth, K., D.M. Lau, F.R. Bischoff, M. Kunzler, E. Hurt, and G. Simos. 1998. Yeast Loslp has properties of an exportinlike nucleocytoplasmic transport factor for tRNA. Mol. Cell. Biol. 18: 6374-6386.

Hopper, A.K. and N.C. Martin. 1992. Processing of yeast cytoplasmic and mitochondrial precursor tRNAs. In The molecular and cellular biology of the yeast Saccharomyces: Gene expression (ed. E.W. Jones, J.R. Pringle, and J.R. Broach), pp. 99-141. Cold Spring Harbor Laboratory Press, Cold Spring Harbor, NY

Hopper, A.K., F. Banks, and V. Evangelidis. 1978. A yeast mutant which accumulates precursor tRNAs. Cell 14: 211-219.

Hopper, A.K., L.D. Schultz, and R.A. Shapiro. 1980. Processing of intervening sequences: A new yeast mutant which fails to excise intervening sequences from precursor tRNAs. Cell 19: $741-751$.

Hunter, L.A., A.L. Benko, J.P. Aris, D.R. Stanford, N.C. Martin, and A.K. Hopper. 1999. S. cerevisiae Mod5p-II contains sequences antagonistic for nuclear (nucleolar) and cytosolic locations. Genetics (in press).

Hurt, D.J., S.S. Wang, Y.H. Lin, and A.K. Hopper. 1987. Cloning and characterization of LOS1, a Saccharomyces cerevisiae 
gene that affects tRNA splicing. Mol. Cell. Biol. 7: 12081216.

Izaurralde, E. and S. Adam. 1998. Transport of macromolecules between the nucleus and the cytoplasm. RNA 4: 351-364.

Izaurralde, E., U. Kutay, C. von Kobbe, I.W. Mattaj, and D. Görlich. 1997. The asymmetric distribution of the constituents of the Ran system is essential for transport into and out of the nucleus. EMBO J. 16: 6535-6547.

Jacobson, M.R., L.-G. Cao, K. Taneja, R.H. Singer, Y. Wang, and T. Pederson. 1997. Nuclear domains of the RNA subunit of RNase P. J. Cell Sci. 110: 829-837.

Kerjan, P., C. Cerini, M. Semeriva, and M. Mirande. 1994. The multienzyme complex containing nine aminoacyl-tRNA synthetases is ubiquitous from Drosophila to mammals. Biochem. Biophys. Acta 1199: 293-297.

Klebe, C., F.R. Bischoff, H. Ponstingl, and A. Wittinghofer. 1995. Interaction of the nuclear GTP-binding protein Ran with its regulatory proteins RCC1 and RanGAP1. Biochemistry 34: 639-647.

Knapp, G., J.S. Beckmann, P.F. Johnson, S.A. Fuhrman, and J. Abelson. 1978. Transcription and processing of intervening sequences in yeast tRNA genes. Cell 14: 221-236.

Knapp, G., R.C. Ogden, C.L. Peebles, and J. Abelson. 1979. Splicing of yeast tRNA precursors: Structure of the reaction intermediates. Cell 18: 37-45.

Kutay, U., G. Lipowsky, E. Izaurralde, F.R. Bischoff, P. Schwarzmaier, E. Hartmann, and D. Görlich. 1998. Identification of a tRNA-specific nuclear export receptor. Mol. Cell 1: 359369.

Lee, B., A.G. Matera, D.C. Ward, and J. Craft. 1996. Association of RNase mitochondrial RNA processing enzyme with ribonuclease P in higher ordered structures in the nucleolus: A possible coordinate role in ribosome biogenesis. Proc. Natl. Acad. Sci. 93: 11471-11476.

Lee, J-Y., C.E. Rohlman, L.A. Molony, and D.R. Engelke. 1991. Characterization of RPR1, an essential gene encoding the RNA component of Saccharomyces cerevisiae nuclear RNase P. Mol. Cell. Biol. 11: 721-730.

Li, Z. and M.P. Deutscher. 1996. Maturation pathways for E. coli tRNA precursors: A random multienzyme pathway in vivo. Cell 86: 503-512.

Lin-Marq, N. and S.G. Clarkson. 1998. Efficient synthesis, termination and release of RNA polymerase III transcripts in Xenopus extracts depleted of La protein. EMBO J. 17: 20332041.

Lund, E. and J.E. Dahlberg. 1998. Proofreading and aminoacylation of tRNAs prior to export from the nucleus. Science (in press).

Maden, B.E.H. 1998. Intracellular locations of RNA-modifying enzymes. In Modification and editing of RNA (ed. $\mathrm{H}$. Grosjean and R. Benne), pp. 421-440. American Society for Microbiology, Washington, D.C.

Matera, A.G., M.R. Frey, K. Margelot, and S.L. Wolin. 1995. A perinucleolar compartment contains several RNA polymerase III transcripts as well as the polypyrimidine tract-binding protein, hnRNP I. J. Cell Biol. 129: 1181-1193.

Mattaj, I.W. and L. Englmeier. 1998. Nucleocytoplasmic transport: The soluble phase. Annu. Rev. Biochem. 67:265-306.

Melton, D.A., E.M. De Robertis, and R. Cortese. 1980. Order and intracellular location of the events involved in the maturation of a spliced tRNA. Nature 284: 143-148.

Morrissey, J.P. and D. Tollervey. 1995. Birth of the snoRNPs: The evolution of RNase MRP and the eukaryotic pre-rRNAprocessing system. Trends Biochem. Sci. 20: 78-82.

Motorin, Y., G. Keith, C. Simon, D. Foiret, G. Simos, E. Hurt, and H. Grosjean. 1998. The yeast tRNA:pseudouridine syn- thase Puslp displays a multisite substrate specificity. $R N A$ 4: 856-869.

Nishikura, K. and E.M. De Robertis. 1981. RNA processing in microinjected Xenopus oocytes. Sequential addition of base modifications in a spliced transfer RNA. I. Mol. Biol. 145: 405-420.

O'Connor, J.P. and C.L. Peebles. 1991. In vivo pre-tRNA processing in Saccharomyces cerevisiae. Mol. Cell. Biol. 11: 425-439.

Peebles, C.L., P. Gegenheimer, and J. Abelson. 1983. Precise excision of intervening sequences from precursor tRNAs by a membrane-associated yeast endonuclease. Cell 32: 525536.

Popenko, V.I., J.L. Inavova, N.E. Cherny, V.V. Filonenko, S.F. Beresten, A.D. Wolfson, and L.L. Kisselev. 1994. Compartmentalization of certain components of the protein synthesis apparatus in mammalian cells. Eur. J. Cell Biol. 65: 6069.

Quevillon, S., F. Agou, J.-C. Robinson, and M. Mirande. 1997. The p43 component of the mammalian multi-synthetase complex is likely to be the precursor of the endothelial monocyte-activating polypeptide II cytokine. J. Biol. Chem. 272: 32573-32579.

Rauhut, R., P.R. Green, and J. Abelson. 1990. Yeast tRNA-splicing endonuclease is a heterotrimeric enzyme. J. Biol. Chem. 265: 18180-18184.

Rose, A.M., H.G. Belford, W.C. Shen, C.L. Greer, A.K. Hopper, and N.C. Martin. 1995. Location of $\mathrm{N}^{2}, \mathrm{~N}^{2}$-dimethylguanosine -specific tRNA methyltransferase. Biochimie 77: 45-53.

Sarkar, S. and A.K. Hopper. 1998. tRNA nuclear export in $S$. cerevisiae: In situ hybridization analysis. Mol. Biol. Cell 9: 3041-3055.

Sharma, K., E. Fabre, H. Tekotte, H.C. Hurt, and D. Tollervey. 1996. Yeast nucleoporin mutants are defective in pre-tRNA splicing. Mol. Cell. Biol. 16: 294-301.

Simos, G., A. Segref, F. Fasiolo, K. Hellmuth, A. Shevchenko, M. Mann, and E.C. Hurt. 1996a. The yeast Arc1p binds to tRNA and functions as a cofactor for the methionyl- and glutamylsynthetases. EMBO I. 15: 5437-5448.

Simos, G., H. Tekotte, H. Grosjean, A. Segref, K. Sharma, D. Tollervey, and E.C. Hurt. 1996b. Nuclear pore proteins are involved in the biogenesis of functional tRNA. EMBO $\mathrm{J}$. 15: 2270-2284.

Simos, G., A. Sauer, F. Fasiolo, and E.C. Hurt. 1998. A conserved domain within Arclp delivers tRNA to aminoacyl-tRNA synthetases. Mol. Cell 1: 235-242.

Spinelli, S.L., S.A. Consaul, and E.M. Phizicky. 1997. A conditional lethal yeast phosphotransferase (tpt1) mutant accumulates tRNAs with a 2'-phosphate and an undermodified base at the splice junction. RNA 3: 1388-1400.

Stapulionis, R. and M.P. Deutscher. 1995. A channeled tRNA cycle during mammalian protein synthesis. Proc. Natl. Acad. Sci. 92: 7158-7161.

Stolc, V. and S. Altman. 1997. Rpp1, an essential protein subunit of nuclear RNase P required for processing of precursor tRNA and 35S precursor rRNA in Saccharomyces cerevisiae. Genes \& Dev. 11: 2926-2937.

Tanner, N.K., M.M. Hanna, and J. Abelson. 1988. Binding interactions between yeast tRNA ligase and a precursor transfer ribonucleic acid containing two photoreactive uridine analogues. Biochemistry 27: 8852-8861.

Tobian, J.A., L. Drinkard, and M. Zasloff. 1985. tRNA nuclear transport: Defining the critical regions of human tRNA $\mathrm{i}_{\text {met }}^{\mathrm{i}}$ by point mutagenesis. Cell 43: 415-422.

Traboni, C., G. Ciliberto, and R. Cortese. 1984. Mutations in Box B of the promoter of a eucaryotic tRNA ${ }^{\text {Pro }}$ gene affect 
rate of transcription, processing, and stability of the transcripts. Cell 36: 179-187.

Trotta, C.R., F. Miao, E.A. Arn, S.W. Stevens, C.K. Ho, R. Rauhut, and J.N. Abelson. 1997. The yeast tRNA splicing endonuclease: A tetrameric enzyme with two active site subunits homologous to the archael tRNA endonucleases. Cell 89: 849-858.

Van Horn, D.J., C.J. Yoo, D. Xue, H. Shi, and S.L. Wolin. 1997. The La protein in Schizosaccharomyces pombe: A conserved yet dispensable phosphoprotein that functions in tRNA maturation. RNA 3: 1434-1443.

van Tol, H. and H. Beier. 1988. All human tRNA ${ }^{\text {Tyr }}$ genes contain introns as a prerequisite for pseudouridine biosynthesis in the anticodon. Nucleic Acids Res. 16: 1951-1966.

Weis, K. 1998. Importins and exportins: How to get in and out of the nucleus. Trends Biochem. Sci. 23: 185-189.

Westaway, S.K. and J. Abelson. 1995. Splicing of tRNA precursors. In $t R N A$ : Structure, biosynthesis, and function (ed. D. Soll and U.L. RajBhandary), pp. 79-92. American Society for Microbiology Press, Washington, D.C.

Wolfe, C.L., A.K. Hopper, and N.C. Martin. 1996. Mechanisms leading to and the consequences of altering the normal distribution of $\mathrm{ATP}(\mathrm{CTP}):$ tRNA nucleotidyltransferase in yeast. J. Biol. Chem. 271: 4679-4686.

Yoo, C.J. and S.L. Wolin. 1997. The yeast La protein is required for the $3^{\prime}$ endonucleolytic cleavage that matures tRNA precursors. Cell 89: 393-402.

Zasloff, M., M. Rosenberg, and T. Santos. 1982. Impaired nuclear transport of a human variant tRNA $\mathrm{i}_{\text {Met }}^{\mathrm{i}}$. Nature 300: $81-84$. 


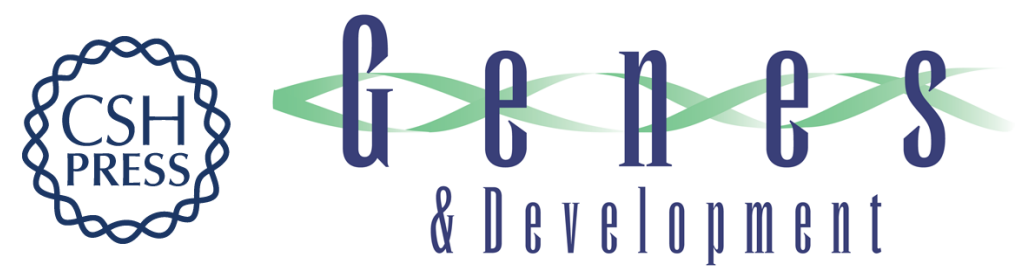

\section{The trials and travels of tRNA}

Sandra L. Wolin and A. Gregory Matera

Genes Dev. 1999, 13:

Access the most recent version at doi:10.1101/gad.13.1.1

References This article cites 70 articles, 35 of which can be accessed free at: http://genesdev.cshlp.org/content/13/1/1.full.html\#ref-list-1

License

Email Alerting Receive free email alerts when new articles cite this article - sign up in the box at the top Service right corner of the article or click here.

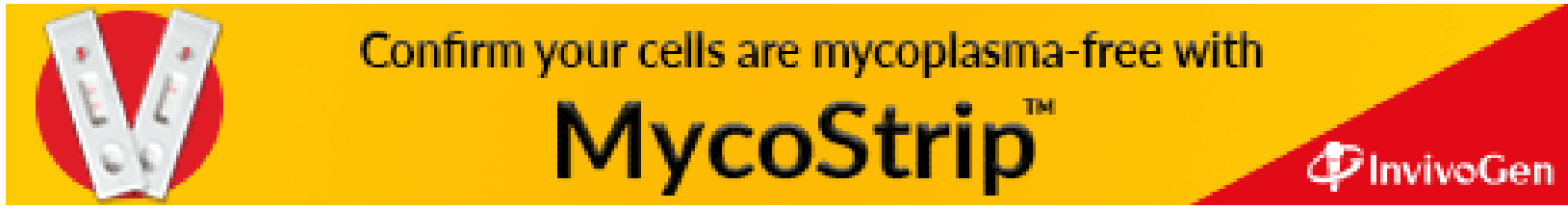

